\title{
Parasitóides de dípteros coletados em um matadouro de Tupaciguara, Minas Gerais, Brasil
}

\section{Parasitoids of dipterous collected in a slaughterhouse of Tupaciguara, Minas Gerais, Brazil}

\section{Carlos Henrique Marchiori ${ }^{1 *}$; Luiz Fernando Barbaresco ${ }^{2}$; Michelle Fernandes Miranda ${ }^{2}$}

\section{Resumo}

Espécies de parasitóides associados com dípteros sinantrópicos foram coletados em fígado bovino no matadouro de Tupaciguara, Estado de Minas Gerais, de março a dezembro de 2005. As pupas foram obtidas pelo método de flotação. Elas foram individualizadas em cápsulas de gelatina até a emergência dos adultos de dípteros ou de seus parasitóides. A porcentagem total de parasitismo foi $14,1 \%$. Os parasitóides Brachymeria podagrica (Fabricius) (Hymenoptera: Chalcididae), Hemencyrtus sp. (Hymenoptera: Encyrtidae), Nasonia vitripennis (Walker) (Hymenoptera: Pteromalidae), Neralsia sp. (Hymenoptera: Figitidae), Pachycrepoideus vindemmiae (Rondani) (Hymenoptera: Pteromalidae) e Spalangia endius Walker (Hymenoptera: Pteromalidae) apresentaram uma porcentagem de parasitismo de $0,6 \%, 0,7 \%, 0,8 \%, 0,1 \%, 11,6 \%$ e $0,3 \%$, respectivamente.

Palavras-chave: Hymenoptera, díptera, armadilha, isca, controle biológico

\begin{abstract}
Species of parasitoids associated with synanthropic dipterous were collected in bovine liver, in a slaughterhouse in Tupaciguara, State of Minas Gerais, from March to December 2005. The pupae were obtained by the flotation method. They were individually placed in gelatin capsules until the emergency of the adult dipterous or their parasitoids. The overall percentage of parasitism was $14.1 \%$. The parasitoids Brachymeria podagrica (Fabricius) (Hymenoptera: Chalcididae), Hemencyrtus sp. (Hymenoptera: Encyrtidae), Nasonia vitripennis (Walker) (Hymenoptera: Pteromalidae), Neralsia sp. (Hymenoptera: Figitidae), Pachycrepoideus vindemmiae (Rondani) (Hymenoptera: Pteromalidae) and Spalangia endius Walker (Hymenoptera: Pteromalidae) presented a parasitism percentage of $0.6 \%, 0.7 \%, 0.8 \%, 0.1 \%, 11.6 \%$ and $0.3 \%$, respectively.

Key words: Hymenoptera, diptera, trap, bait, biocontrol
\end{abstract}

\footnotetext{
1 Biólogo, Doutor em Ciências Biológicas; Prof. do Centro Federal de Educação Tecnológica de Urutaí-UNED-Morrinhos. E-mail: chmarchiori@yahoo.com.br

2 Alunos de Iniciação Científica do Instituto Luterano de Ensino Superior de Itumbiara-ILES-ULBRA.

* Autor para correspondência
} 
Algumas espécies de dípteros das famílias Calliphoridae, Muscidae e Sarcophagidae são importantes nas áreas médica e veterinária, uma vez que podem produzir miíases e atuar na veiculação de patógenos ao homem e aos animais (GREENBERG, 1971). Os dípteros têm sido encontrados transmitindo mais de 100 espécies de organismos causadores de doenças como bactérias, protozoários e helmintos (GREENBERG, 1971). Na zona rural, podem acarretar diminuição da produção de ovos, doenças gastrointestinais e parasitoses nos animais, além de causar incômodo à população humana vizinha às criações (BERTI FILHO; THOMAZINI; COSTA, 1996; MARICONI; GUIMARÃES; BERTI FILHO, 1999).

Para controle dos insetos, podem ser utilizados os chamados reguladores naturais, tanto na agricultura como em áreas de criação animal como os parasitóides (MARCHIORI; OLIVEIRA; LINHARES, 2001; SILVEIRA et al., 1989). Os parasitóides de dípteros são considerados bioindicadores da biodiversidade dos ecossistemas como o cerrado, sendo considerados importantes para a manutenção do equilíbrio das comunidades em que vivem (SCATOLINI; DIAS, 1997).

Os principais gêneros de parasitóides de dípteros pertencem às famílias Braconidae (Aphaereta e Gnathopleura), Chalcididae (Brachymeria), Encrytidae (Hemencyrtus), Figitidae (Neralsia) e Pteromalidae (Nasonia, Pachycrepoideus e Spalangia) (MARCHIORI et al., 2007). A importância dos Hymenoptera Parasitica deriva do efeito regulador que exercem sobre as populações de insetos hospedeiros, graças à grande diversidade de adaptações fisiológicas e comportamentais. Sua importância ecológica e econômica, aliada à falta de conhecimento do grupo, justificou o desenvolvimento deste estudo.

O objetivo desse trabalho é relatar os parasitóides de dípteros coletados no matadouro de Tupaciguara, Minas Gerais.

O estudo foi realizado no Matadouro Municipal, localizado no município de Tupaciguara, MG (18³5’S
- 48 37 W), no período de março a dezembro de 2005. Os dípteros foram coletados quinzenalmente com o uso de armadilhas confeccionadas com recipiente metálicos, medindo cerca de $19 \mathrm{~cm}$ de altura por $9 \mathrm{~cm}$ de diâmetro, de cor preta fosca, com duas aberturas tipo venezianas, confeccionadas no terço inferior, para permitir a entrada dos insetos. Na parte superior da armadilha, foi acoplado um funil de papel com a base voltada para baixo e envolvido em um saco plástico, utilizado para a coleta dos dípteros e parasitóides atraídos (Figura 1) (FERREIRA, 1978). Utilizou-se como isca, fígado bovino, depositado no interior dos recipientes metálicos, sobre uma camada de terra. Empregaramse 6 armadilhas, as quais foram penduradas duas nos currais, duas nas pocilgas dos suínos, uma no local onde são armazenados os restos de ossos e uma na sala de couro. Os indivíduos adultos coletados foram levados para o laboratório, mortos com éter etílico e conservados em álcool 70\% para posterior identificação.

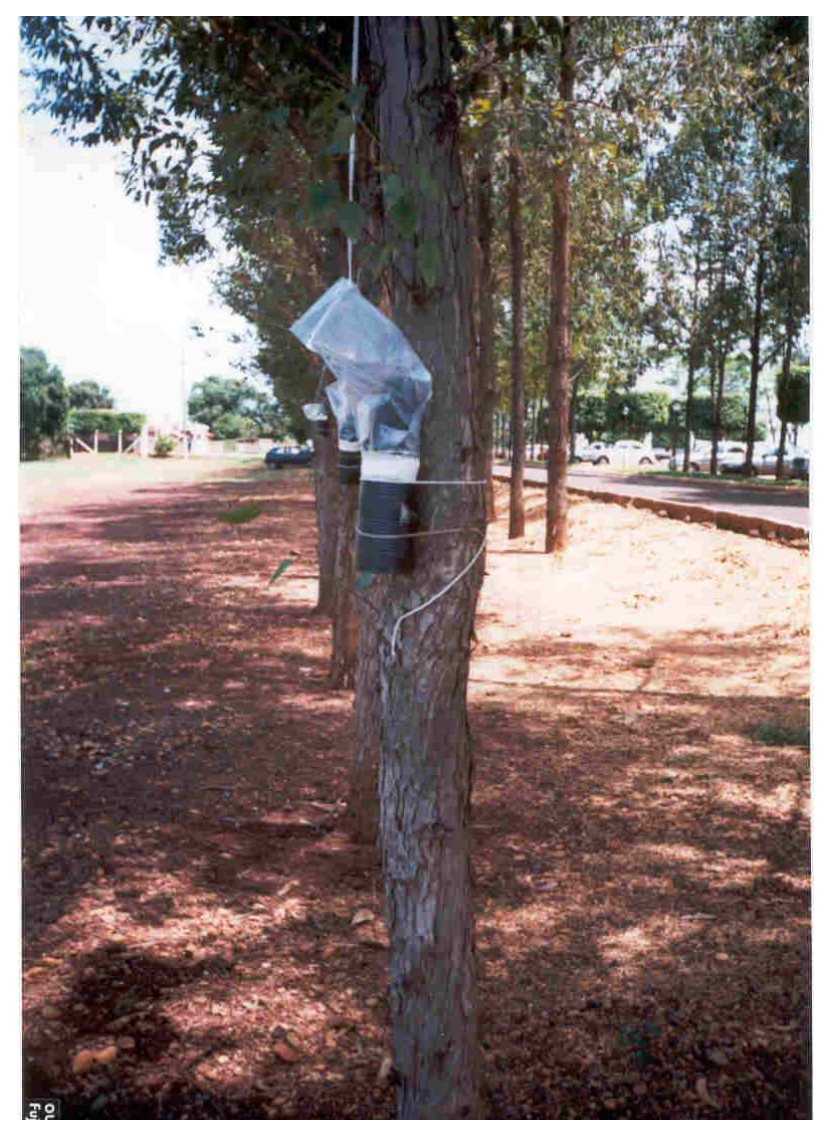

Figura 1. Aspecto geral da armadilha 
Tendo em vista uma eventual oviposição dos insetos atraídos, o conteúdo das armadilhas foi colocado em recipientes plásticos, contendo uma camada de areia para servir de substrato à pupação das larvas. Após 15 dias da colocação das armadilhas no campo, a areia foi peneirada e dela se recolheram as pupas, que, em seguida, foram individualizadas em cápsulas de gelatina (número 00) para obtenção dos dípteros e/ou parasitóides.

Os parasitóides e os dípteros foram identificados por um dos autores. A hipótese de atração dos parasitóides pelos seus hospedeiros foi testada pelo Qui-Quadrado, ao nível de 5\% de probabilidade.

Obtiveram-se 1652 pupários de dípteros, dos quais emergiram 411 espécimes de parasitóides (Tabela
1), sendo 10 exemplares de Brachymeria podagrica (Fabricius) (Hymenoptera: Chalcididae), 75 exemplares de Hemencyrtus sp. (Hymenoptera: Encyrtidae), 128 exemplares de Nasonia vitripennis (Walker) (Hymenoptera: Pteromalidae), um de Neralsia sp. (Hymenoptera: Figitidae), 192 exemplares de Pachycrepoideus vindemmiae (Rondani) (Hymenoptera: Pteromalidae) e cinco exemplares de Spalangia endius Walker (Hymenoptera: Pteromalidae). O fato de existir um matadouro muito próximo da mata, contribuiu para a coleta dos parasitóides e dos dípteros. As áreas de mata são importantes como locais de origem de parasitóides, os quais são inimigos naturais de outros insetos.

Tabela 1. Parasitóides e seus dípteros coletados no Matadouro de Tupaciguara, Minas Gerais, no período de março a dezembro de 2005.

\begin{tabular}{|c|c|c|c|c|}
\hline Grupo taxonômico & Freqüência & $\begin{array}{l}\text { Espécies de } \\
\text { parasitóides }\end{array}$ & Frequiência & Número de pupas parasitadas \\
\hline \multicolumn{5}{|l|}{ Calliphoridae: } \\
\hline Chrysomya albiceps & 74 & Hemencyrtus sp. & 20 & 3 \\
\hline \multicolumn{5}{|l|}{ Fanniidae: } \\
\hline Fannia pusio & 428 & P. vindemmiae & 64 & 64 \\
\hline \multicolumn{5}{|l|}{ Muscidae: } \\
\hline \multirow[t]{3}{*}{ Musca domestica } & 392 & Hemencyrtus sp. & 35 & 5 \\
\hline & & $P$. vindemmiae & 63 & 63 \\
\hline & & S. endius & 4 & 4 \\
\hline Ophyra sp. & 64 & Hemencyrtus sp. & 20 & 3 \\
\hline \multicolumn{5}{|l|}{ Phoridae: } \\
\hline Megaselia scalaris & 492 & P. vindemmiae & 63 & 63 \\
\hline \multicolumn{5}{|l|}{ Sarcophagidae: } \\
\hline \multirow[t]{2}{*}{ Oxysarcodexia thornax } & 139 & B. podagrica & 8 & 8 \\
\hline & & Neralsia sp. & 1 & 1 \\
\hline \multirow[t]{3}{*}{ Peckia chrysostoma } & 40 & N. vitripennis & 82 & 4 \\
\hline & & P. vindemmiae & 2 & 2 \\
\hline & & S. endius & 1 & 1 \\
\hline \multirow[t]{2}{*}{ Sarcodexia lambens } & 23 & B. podagrica & 2 & 2 \\
\hline & & N. vitripennis & 12 & 1 \\
\hline Total & 1652 & - & 411 & 233 \\
\hline
\end{tabular}

Entre os dípteros, Megaselia scalaris (Loew) (Diptera: Phoridae) foi a espécie mais coletada, com $29,8 \%$, sendo encontrada em vários ambientes, além de ela ter capacidade de explorar uma grande variedade de nichos ecológicos. As larvas podem causar miĺases em diferentes animais e no homem (CARVALHO et al., 2000; ROBINSON, 1975). 
Dos dípteros coletados, os mais importantes foram as espécies Chrysomya albiceps (Wiedennam) (Diptera: Calliphoridae) e Musca domestica L. (Diptera: Muscidae). C. albiceps é uma espécie de importância médica, por ser produtora de miíases secundárias e apresenta significativo papel como predadora de outras larvas de dípteros. Por outro lado, esse díptero assume uma relevante importância em Saúde Pública, por ser veiculadora mecânica de microrganismos patogênicos para o homem. $M$. domestica é a espécie de maior interesse sanitário, devido a seu caráter sinantrópico, endofilia, sua abundância na região urbana, capacidade de se desenvolver em vários tipos de substratos, seu alto poder reprodutivo e de ser apontada como veiculadora de patógenos ao homem e animais (D’ALMEIDA, 1986; GREENBERG, 1971).

Provavelmente, a presença dos dípteros coletados está correlacionada com o local onde foram colocadas as armadilhas.

Em relação aos parasitóides, $P$. vindemmiae foi a espécie mais coletada, com $82,4 \%$ dos indivíduos coletados, provavelmente devido ao fator sazonalidade ou à capacidade de busca apresentada pela espécie, que é considerada um parasitóide solitário de numerosos dípteros das famílias Anthomyiidae, Calliphoridae, Muscidae, Sarcophagidae, Tachinidae e Tephritidae. P. vindemmiae apresenta ampla distribuição geográfica, sendo também encontrada na América do Norte e África (HANSON; GAULD, 1995).

A porcentagem total de parasitismo foi de $14,1 \%$. A porcentagem total de parasitismo apresentada pelos parasitóides B. podagrica, Hemencyrtus sp., $N$. vitripennis, Neralsia sp., $P$. vindemmiae e $S$. endius foi de $0,6 \%, 0,7 \%, 0.8 \%, 0,1 \%, 11,6 \%$ e $0,3 \%$, respectivamente. A maior porcentagem de parasitismo ocorreu com $P$. vindemmiae, provavelmente pelo seu hábito polífago.

A espécie $P$. vindemmiae apresentou maior porcentagem de parasitismo em $M$. domestica, com $16,1 \%$, provavelmente pela habilidade competitiva das formas jovens. Essa espécie também parasitou uma maior diversidade de espécies de dípteros. $\mathrm{O}$ fato de utilizar muitos dípteros, $P$. vindemmiae, favorece sua permanência no meio ambiente, aumentando o seu potencial como agente no controle biológico.

Ainda em relação à atração dos parasitóides pelos dípteros, verificou-se que $B$. podagrica apresentou atração por Oxysarcodexia thornax (Walker) e Sarcodexia lambens (Wiedemann) (Diptera: Sarcophagidae); Hemencyrtus sp. por C. albiceps, M. domestica e Ophyra sp. (Diptera: Muscidae); $N$. vitripennis por $C$. albiceps, Peckia chrysostoma (Wiedemann) (Diptera: Sarcophagidae) e S. lambens; P. vindemmiae por Fannia pusio (Diptera: Fanniidae), M. domestica e $M$. scalaris; Neralsia sp. por O. thornax e $S$. endius por $M$. domestica e $P$. chrysostoma $\left(\mathrm{X}^{2}=471,08\right.$; $\mathrm{GL}=35 ; \mathrm{P}<0,05)$.

Espera-se com esse trabalho, ampliar os conhecimentos sobre a fauna de dípteros sinantrópicas e seus parasitóides e identificar possíveis espécies que possam ser utilizadas em futuros programas de controle biológico.

\section{Referências}

BERTI FILHO, E.; THOMAZINI, M. J.; COSTA, V. A. Artrópodes benéficos associados ao esterco de galinhas poedeiras no Brasil. Revista de Agricultura, Piracicaba, v.7, n.3, p.273-286, 1996.

CARVALHO, L. M. L.; THYSSEN, P. J.; LINHARES, A. X.; PALHARES, F. A. B. A checklist of arthropods associated with pig carrion and human corpses in Southeastern Brazil. Memórias do Instituto Oswaldo Cruz, Rio de Janeiro, v.95, n.1, p.135-138, 2000.

D’ALMEIDA, J. M. Substratos utilizados para a criação de dípteros caliptratos em uma área rural do Estado do Rio de Janeiro. Arquivo da Universidade Federal Rural do Rio de Janeiro, Rio de Janeiro, v.9, p.13-22, 1986.

FERREIRA, M. J. M. Sinantropia de dípteros muscóideos de Curitiba, Paraná: Calliphoridae. Revista Brasileira de Biologia, São Carlos, v.38, n.2, p.445-454,1978.

GREENBERG, B. Flies and disease: ecology, classification and biotic association. New Jersey: Princeton University Press, 1971. 
HANSON, P. E.; GAULD, I. D. The hymenoptera of Costa Rica. Oxford: University Press Oxford, 1995.

MARCHIORI, C. H.; OLIVEIRA, A. T.; LINHARES, A. X. Artrópodes associados a massas fecais bovinas no Sul do Estado de Goiás. Neotropical Entomology, Londrina, v.30, n.1, p.19-24, 2001.

MARCHIORI, C. H.; SILVA FILHO, O. M; BORGES, M. P.; ALVARENGA, A. V. Parasitóides de dípteros (Insecta: Diptera) coletados usando armadilha ptifall em Itumbiara, Goiás. Biotemas, Florianópolis, v.20, n.1, p.115-118, mar. 2007.

MARICONI, F. A. M.; GUIMARÃES, J. H.; BERTI FILHO, E. A mosca doméstica. Piracicaba: FEALQ. 1999.
ROBINSON, W. H. Megaselia (M.) scalaris (Diptera: Phoridae) associated with laboratory cockroach colonies. Proceedings of the Entomological Society of Washington, Washington, v.77, p.384-390, 1975.

SCATOLINI, D.; DIAS, A. M. P. A fauna de Braconidae (Hymenoptera) como bioindicadora do grau de preservação de duas localidades do Estado do Paraná. Revista Brasileira de Ecologia, São Paulo, v.1, p.84-87, 1997.

SILVEIRA, G. A. R.; MADEIRA, N. G.; AZEREDO-ESPIN, A. M. L.; PAVAN, C. Levantamento de microhimenópteros parasitóides de dípteros de importância médico-veterinária no Brasil. Memórias do Instituto Oswaldo Cruz, Rio de Janeiro, v.84, Supl. 4, p.505-510, 1989. 
